\title{
Surgery of benign thyroid disease - Analysis of vocal fold paralysis in 3019 patients
}

\author{
B. Kohnen ${ }^{1}$, C. Schürmeyer ${ }^{2}$, T. H. Schürmeyer ${ }^{2}$, P. Kress ${ }^{1}$
}

Departments of ${ }^{1}$ ENT and ${ }^{2}$ Internal Medicine II, Klinikum Mutterhaus der Borromäerinnen, Trier, Germany

\section{INTRODUCTION}

Thyroid gland surgery is one of the most frequent elective surgical procedures in Germany. In 201477093 (1) thyroid surgeries (total thyroidectomy, hemi-thyroidectomy and partial thyroidectomy) were performed. The main complications are injury of the recurrent laryngeal nerve with permanent or transient vocal fold palsy, permanent or transient hypocalcaemia, infections and postoperative bleeding. To evaluate factors influencing the relative risk (RR) for vocal fold paralysis we analysed all patients who had a surgery for benign thyroid diseases in the Klinikum Mutterhaus der Borromäerinnen from 2001-2014.

\section{METHODS}

In a retrospective study the data of 3019 patients were analyzed. Operations were performed with intraoperative neuromonitoring by general surgeons (GS) $(n=1637)$ or physicians specially trained for ENT/Head and Neck Surgery $(n=1382) .19,2 \%$ of the procedures were carried out by residents in training. The following information was considered: gender, age, type of operation, technique (minimally invasive (MIVAT) vs. conventional), executing department, experience of the surgeon, indication of surgery, revision procedure and complications (hypocalcaemia, postoperative bleeding, vocal cord palsy). Every patient had an endoscopic examination of the larynx by an ENT-specialist during the inpatient stay to detect vocal fold paralysis. No discrimination was made between permanent and transient paralysis due to the lack of follow up laryngoscopy.

\section{RESULTS}

The main indications for surgery were nodular goiter (58.7\%), inactive nodes (17.5\%), thyroid autonomy (16.3\%) or Grave's disease (6.5\%). Vocal fold paralysis occurred in 198 subjects (6.6\%), $6.3 \%$ in females and $7.0 \%$ in males. Prevalence was not influenced by gender, age, recurrent disease ( $R R$ 1.20), surgeries performed by physicians in training $(6.2 \%, n=581)$, or if minimal invasive $(6.5 \%, n=769)$ and conventional surgery $(6,6 \%, n=2250)$ were compared. Risk was increased in nodular goiter $(7.3 \%, R R$ 1.37), but not in Grave's disease (6.6\%, $R R$ 1.01). A higher rate was seen in total $(n=1576,6.9 \%)$ and nearly total $(n=507,8.1 \%)$, than in subtotal thyroidectomy $(n=866,5.2 \%)$. A lower rate (RR 0.54) was observed in operations performed by ENT (4.6\%), than by GS (8.2\%). Postoperative haemorrhage (4.6\% vs. $1.6 \%, R R 2.94)$, hypocalcemia $<2,0 \mathrm{mmol} / \mathrm{l}(28.8 \%$ vs. $19.9 \%, R R 1.63)$ and the need for calcium substitution ( $R R$ 1.65) or dihydrotachysterol treatment (RR 2.18) at discharge from the hospital was documented more frequently in patients with recurrent nerve palsy.

\section{CONCLUSION}

In thyroid surgery a multinodular goiter, invasiveness of the operations and a physician not trained for head and neck surgery are risk factors for vocal fold paralysis. Postoperative haemorrhage and hypocalcemia are seen more frequently in patients with damage to the recurrent laryngeal nerve.

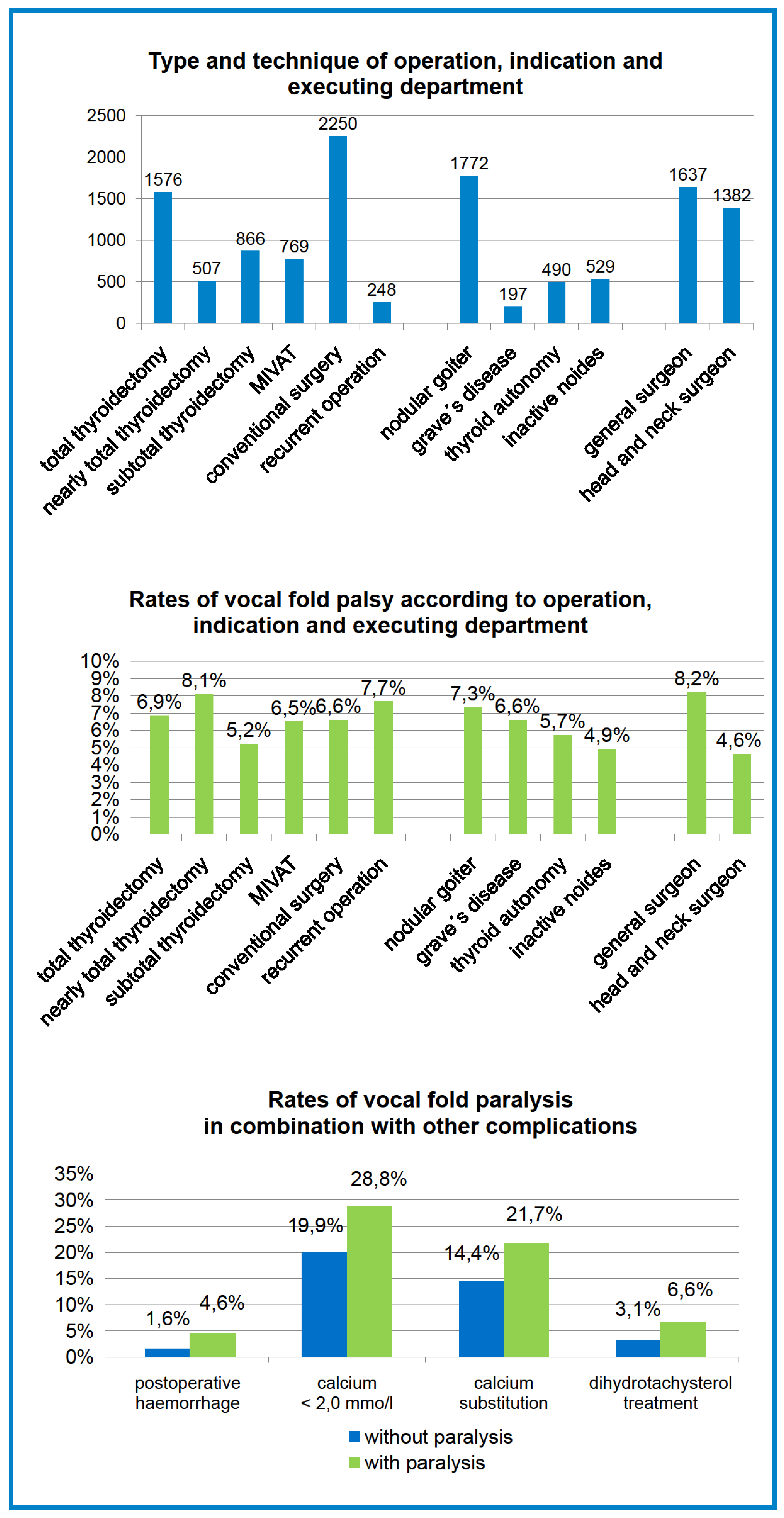

REFERENCES

(1) Gesundheitsberichterstattung des Bundes -www.gbe-bund.de

\section{KLINIKUM}

MUTTERHAUS DER BORROMÄERINNEN 